\section{BIOMARKER CORRELATES OF RESPONSE IN PATIENTS WITH ADVANCED MYXOID/ROUND CELL LIPOSARCOMA (MRCLS) TREATED WITH NY-ESO-1 TCR T CELLS (LETETRESGENE AUTOLEUCEL)}

${ }^{1}$ Gurpreet Kapoor*, 'Stefan Zajic, 'Sunil Suchindran, 'Jaegil Kim, 'Ioanna Eleftheriadou, ${ }^{1}$ Anne Huff, ${ }^{1}$ Michael Nathenson, ${ }^{2}$ Mihaela Druta, ${ }^{3}$ Brian Van Tine, ${ }^{4}$ Neeta Somaiah, ${ }^{5}$ David Liebner, ${ }^{6}$ Scott Schuetze, ${ }^{7}$ Sandra D'Angelo. ${ }^{1}$ GlaxoSmithKline, Collegeville, USA; ${ }^{2} \mathrm{H}$. Lee Moffitt Cancer Center, Tampa, FL, USA; ${ }^{3}$ Washington University in St. Louis, St Louis, USA; ${ }^{4}$ University of Texas/MD Anderson Cancer Center, Houston, USA; ${ }^{5}$ The Ohio State University, Columbus, OH, USA; ${ }^{6}$ University of Michigan, Ann Arbor, USA; ${ }^{7}$ Memorial Sloan Kettering Cancer Center, New York, USA

Background This is an open label pilot study (NCT02992743) on letetresgene autoleucel (lete-cel; GSK3377794), an NYESO-1-specific autologous CD4+ and CD8+ T cells expressing a high affinity $\mathrm{T}$-cell receptor which recognizes the NYESO-1 antigen epitope in complex with specific HLA- alleles $\mathrm{A}^{* 02}$, which exhibited anti-tumor activity and manageable safety profiles in patients with advanced MRCLS based on interim analysis (IA) data. ${ }^{1}$ Lymphodepletion has been shown to enhance the expansion, persistence, and homing of therapeutically infused T-cells, thereby potentiating therapeutic efficacy against malignant diseases. ${ }^{2}$ Initial T-cell kinetics data from this study demonstrated that lymphodepletion regimen (LDR)-B robustly depleted lymphocytes at infusion and was trended with higher peak cell expansion (Cmax) vs. LDR-A. The peak expansion was significantly associated with weightnormalized transduced cell dose and trended with response. ${ }^{1}$ Here, we will be discussing additional cell kinetics data and other exploratory biomarker correlates of response.

Methods Patients with advanced MRCLS were enrolled to 2 cohorts and received either planned $A(\mathrm{~N}=10)$ or $\mathrm{B}(\mathrm{N}=10)$ LDRs prior to lete-cel infusion (table 1). Response was assessed per RECIST v1.1. Transduced cell kinetics were measured by quantitative PCR of transgene vector copies in DNA from peripheral blood mononuclear cells (PBMCs). Serum cytokines (Meso Scale Discovery immunoassay) as pharmacodynamic (PD) markers of response and their association with $\mathrm{T}$ cell kinetics will be discussed. Phenotypic characterization of the cell product (pre- and post- infusion) via Flow cytometry using Cytek Aurora (23 color panel), to help understand correlation of response with engineered cell product attributes, will be presented. Potential biomarker correlates of clinical response were tested using generalized linear models.

Results Five out of 6 responders with available lab data exhibited robust lymphocyte depletion at infusion $(0-25$ cell $/ \mu \mathrm{L})$ and high Cmax (>50,000 vector copies/ $\mu$ g gDNA) with LDR. Only 6/14 non-responders exhibited low lymphocytes counts at infusion and high Cmax. LDR-B also induced strong depletion of monocytes at infusion $(p=0.03)$ vs. LDR-A, but depletion of monocytes did not show association with response. Higher Cmax was correlated with exposure $\left(\mathrm{AUC}_{0-28 \mathrm{~d}}\right.$ ) (Adj. $\left.\mathrm{R}^{2}=0.606\right)$. $\mathrm{AUC}_{0-28 \mathrm{~d}}$ was a better predictor of response in patients receiving LDR-B ( $\mathrm{p}=0.0182)$, with $\mathrm{AUC}_{0-28 \mathrm{~d}}$ trending towards predicting response in the LDR-A cohort. $\mathrm{AUC}_{0-28 \mathrm{~d}}$ was associated with tumor volume reduction $(p=0.0569)$.

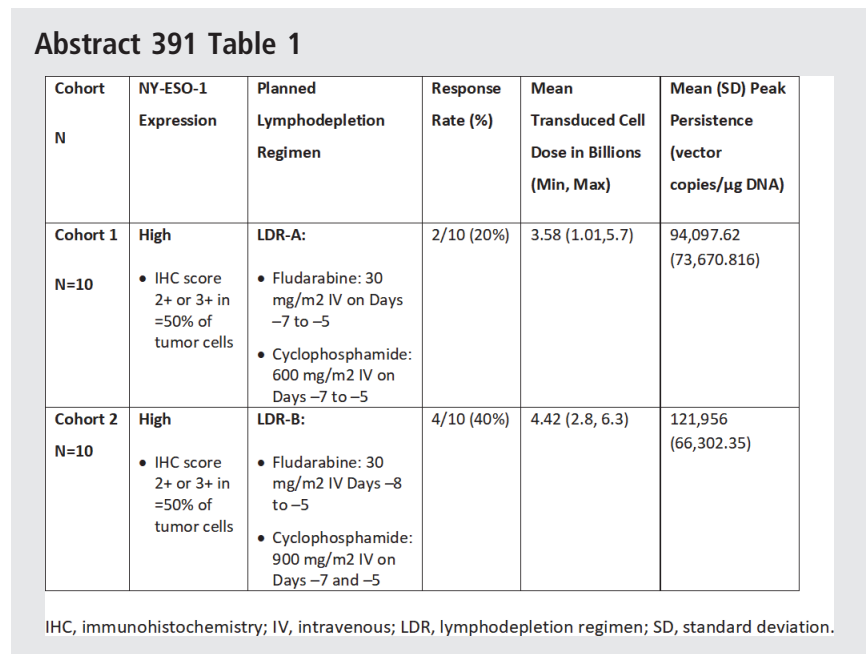

Conclusions Exposure-response analysis of this study reveals that efficacy appears to be driven by weight-normalized transduced cell dose and $\mathrm{LDR}$ via $\mathrm{AUC}_{0-28 \mathrm{~d}}$. Higher $\mathrm{AUC}_{0-28 \mathrm{~d}}$ was correlated with Cmax and maximum tumor volume reduction. Acknowledgements This study (208469; NCT02992743) was funded by GlaxoSmithKline.

Trial Registration NCT02992743

\section{REFERENCES}

1.. D'Angelo SP, et al. J Clin Oncol 2021;39:15_suppl:11521.

2.. Bechman, Maher. Expert Opin Biol Ther 2021;21(5):627-637.

Ethics Approval This study was approved by institutional review boards (IRB) at the six participating sites.

http://dx.doi.org/10.1136/jitc-2021-SITC2021.391 